\title{
Study of pharmacodynamic material basis of single- component Chinese medicine based on metabolomics
}

\author{
Yueling Yuan MSc, Xi Xia MSc, Qianwen Li MSc, Yu Cai PhD
}

Y Yuan, X Xia, Q Li, Y Cai. Study of pharmacodynamic material basis of single-component Chinese medicine based on metabolomics. Curr Res Integr Med 2015;1(3):41-44.

Metabolomics is the holistic qualification and quantification of metabolites in vivo. Metabolomics explores the relative relationship between metabolites and physiological or pathological changes. Its characteristics of integrity, high throughput and the 'end of the end' has a prominent advan- tage in the study of the Chinese medicine overall efficacy and the mechanisms of multitarget effects. The present article briefly summarizes research regarding single-component Chinese medicine efficacy and functional mechanisms based on the science and techology of metabolomics.

Key Words: Chinese medicine; Functional mechanism; Metabolomics

\section{METABONOMIC STUDY OF THE PHARMACODYNAMIC MATERIAL BASIS OF SINGLE HERBS}

TCM has the characteristics of multiple components and multiple targets, and metabonomics provides the possibility to establish the related "composition-activity relationship" by identifying the effective components of Chinese medicine on the whole .

Ding (27) established the fingerprint of the volatile oil from curcuma by using GC-MS, studied the relationship between various volatile oils constituents and antitumor activity, and built the optimal "composition-activity relationship" model. Wu et al (28) analyzed the relationship between Salvia miltiorrhiza components and antioxidant abilities by using the research strategy of the metabonomics, and realized the activity prediction of composition. Chen (29) also used GC-MS technique to analyze chemical constituents of volatile oil of Ligusticum chuanxiong Hort (LCH), data mining the volatile oil chemical composition and relevance of vascular active data, and discriminated the main component of $\mathrm{LCH}$ that has a vasodilator effect. $\mathrm{Li}$ et al (30) based on the least angle regression algorithm, research the relationship between LCH constituents combination and vascular activity and realized the activity optimization of constituents combination. $\mathrm{Li}$ et al (31) described the pharmacometabolomics study investigating the therapeutic basis and metabolic effects of Epimedium brevicornum Maxim on hydrocortisone-induced rat using ultra-performance (UP) LC-MS. The results showed that icariin and epimedin C may be the main pharmacodynamic material basis of Epimedium brevicornum Maxim. Zhang (32) used the composition-activity relationship to identify antitumour compounds from turmeric based on orthogonal partial least squares and compatibility research, and finally obtained seven optimum combinations. Yu et al (33) explored the antibacterial mode of Hemsleya pengxianensis against Staphylococcus aureus. The results revealed the antibacterial mechanism to be similar to vancomycin by inhibiting cell wall synthesis, and found the main antibacterial component is cucurbitacins. In addition, using the same method, they found that magnoflorine is the main antibacterial component of Aquilegia oxysepala Trautv (34), and learned that the antibacterial effect of Radix tinosporae is due to jatrorrhizine and martin (35). Wang et al (36) studied the pharmacodynamic material basis of honey-stirbaked and liquorice-stir-boiled Polygalae radix (PR) by using plant metabonomic technology based on ${ }^{1} \mathrm{H}-\mathrm{NMR}$ and UPLC. Result shows that the processing may change the medicinal properties so as to lead the various pharmacological activities. Che et al (37) and Wang (38) neural network (26).

College of Pharmacy, JiNan University, Guangzhou 510632, China

Correspondence: Dr Yu Cai, College of Pharmacy, JiNan University, 601 Huangpu Aveneu West, Guangzhou, Guangdong 510632, China.

Telephone 18826411201, e-mail jida2468@163.com 


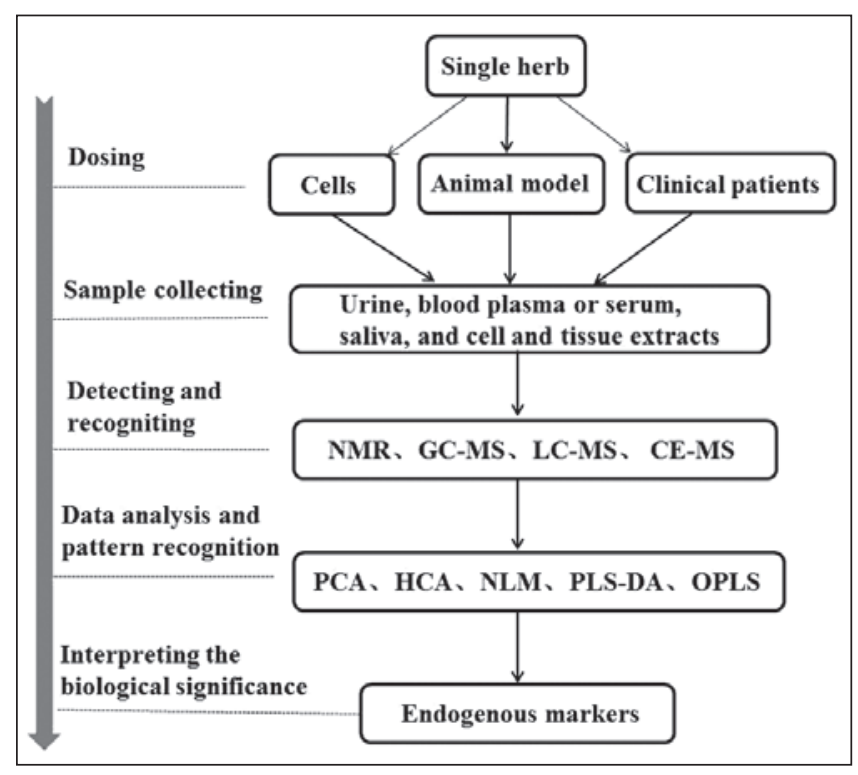

Figure 1) Research content and process of the metabolomics. CE-MS Capillary electrophoresis-mass spectrometry; GC-MS Gas chromatography-mass spectrometry; HCA High content analysis; LC-MS Liqid chromatography-mass spectrometry; NMR Nuclear magnetic resonance; OPLS Orthogonal partial least squares; PCA Principal component analysis; PLS-DA Partial least squares discriminant analysis

have studied the main metabolites in urine and blood of humans and rats that had been dosed with baicalin. They found that baicalein is the main intermediate of drug metabolites, and constitutes the pharmacodynamic material basis of baicalin.

\section{METABONOMIC RESEARCH ON MECHANISMS OF SINGLE CHINESE MEDICINE}

Metabolomics study not only reveals the metabolic processes of TCM, itself with complex characteristics among multicomponents which have interaction, but also speculates the drug-induced change of endogenous metabolites and related pathways to clarify the action of mechanism of a drug.

Lu et al (39) explored the effect of ginsenosides on the metabolism of spontaneously hypertensive rats using GC-MS. Results showed that after the intervention of ginsenosides, the levels of the specific biomarkers - palmitic acid, galactose pyranthrone, linoleic acid and tyrosine - in the blood plasma of spontaneously hypertensive rats had a downward trend and the metabolism returned to normal. Also, the action of mechanism of berberine was confirmed to be associated with the decreased levels of free fatty acid in blood plasma of rats with type I diabetes (40). Meng et al (41), using the metabolomics approach, found eight kinds of potential biomarkers in cold condensation syndrome rats, and the mechanism of action of chuangxiong, a heatnatured medicine, appeared to inhibit the conversion of phosphatidylcholine to phospholipase A2, which produces arachidonic acid ,causing decline in the level of thromboxane synthesis and affecting the metabolic pathways of phosphatidylcholine, thus easin $g$ the symptoms of blood stasis. Yang et al (42), using UPLC-MS, made a comparison of the effects of red peony root and white peony root on toe swelling of rats caused by diagonal carageen glue. Results indicated that endogenous biological markers such as F2 $\alpha$, prostaglandin E3, leukotriene A4, prostaglandin E2 and glutathione play an important role in the anti-inflammatory action of red peony root and white peony root, and lays the foundation for further research on the different mechanisms of action between red peony root and white peony root. Likewise, Li et al (43) used rapid resolution LC-MS, principal component analysis, PLS-DA and other chemometrics methods to select four characteristic endogenous metabolites deriving from chronic renal failure, and found the possibility that rhubarb inhibits the catecholamines generating in the blood of the chronic renal failure rats, the decomposition of phosphate esters and the production of inflammatory mediators, causing the recovery of D-glutamic acid metabolism, D-glutamine metabolism and methionine cycle. Wang et al (44), by using NMR in combination with PLS-DA, analyzed the changes in endogenous metabolites in urine after intraperitoneal injection of allitridum in rats and found that allitridum has an impact on Kreb's cycle and the level of acetone bodies, suggesting that studying this metabonomic interaction contributes to understand the liver-protective mechanism of garlic. By analyzing the change of serum metabolomics in kidney yang deficiency rats induced by hydrocortisone, Morinda officinalis, with an effect of kidney-replenishing, was confirmed to enhance the methyl transfer reaction of the organism's lack of vital energy, improve energy metabolism and reverse amino acid metabolic disorders; this same effect was not seen on intestinal flora (45). Drynaria rhizome, which may associate with the regulation of energy metabolism, metabolism of intestinal bacteria, and amino acid metabolism and antioxidative damage (46), will not only regulate the metabolic disorders in glucocorticoid-induced osteoporosis rats, but also has an effect of kidney-replenishing (47). Using GC-MS metabonomics technology, Wang et al (48) initially demonstrated that Bupleurum chinense DC, with its multiple targets, may participate in enzyme inhibition, neurotransmitters, sugarlipid metabolism, amino acids metabolism, energy metabolism and more aspects to collaboratively exert the antipyretic effect. Gu et al (49) studied the treatment of total ginsenosides to diabetic cardiomyopathy by investigating urine metabolomics and found three kinds of biomarkers, whose mechanism may be that total ginsenosides regulate the citric acid cycle, fatty acid metabolism and oxidative stress. Poria cocos skin, which is possibly involved in the metabolism of phospholipids, energy and amino acids, was found to be effective in regulating six biomarkers, including creatinine, in a study on serum metabonomics of model rats with kidney disease (50). Detoxification with licorice was showed to be associated with the increased free bile acid and bile acid conjugated with glycine in the plasma metabolome of rats (51), whereas the anti-inflammatory action of violet magnolia was speculated to be associated with the metabolism of fatty acids and cholesterol in in the plasma metabolome of rats with inflammation (52). Followed by the metabonomics with LC-MS, Cao (53) analyzed metabolite changes in blood plasma and urine after the interference of total glucosides of peony in acute liver injury rats model induced by $\mathrm{CCl}_{4}$, and detected the retracement of alanine, proline and glycine, suggesting that total glucosides of peony have a preventive effect on $\mathrm{CCl}_{4}$-induced acute liver injury rats model, and it's action mechanism may relate to the Krebs cycle and the synthesis of glutathione eliminating oxygen free radicals.

\section{EXISTING PROBLEMS AND PROSPECTS IN RESEARCH OF METABOLOMICS IN TCM}

Metabolomics shares multitarget, global and systematic principles with with TCM, which validate the unique advantages of the study of efficacy and mechanisms of TCM. Metabonomics has developed rapidly in recent years; however, it is not perfect and there are still some problems and challenges in the modern research of TCM. First, most studies of metabolism are only qualitative analyses rather than quantitative analyses. Second, due to the complexity of metabolites, current technology cannot comprehensively analyze all metabolites in an organism, and differences in concentrations among the metabolites is also a significant challenge for detection sensitivity. Nevertheless, although a variety of data analysis methods have been developed, most are only suitable for linear data and there are some challenges to processing nonlinear data. Finally, the support of complete databases is required by metabonomics (54-56). Metabonomics, with the increasing development and improvement, will fit in the process of modernization and internationalization of TCM in a better way, providing new ideas, new methods and new platform to reveal the mysteries of TCM.

FOUNDATION SUPPORT: The National Natural Science Foundation of China (81173215); the Ministry of Education in the New Century Excellent Talents (NECT-12-0677); 


\section{REFERENCES}

1. Nicholson JK, Lindon JC, Holmes E. "Metabonomic": understanding the metabolic responses of living systems to pathophysiological stimuli via multivariate statistical analysis of biological NMR spectroscopic data. Xenobiotica 1999;29:1181-9.

2. Goodacre R, Vaidyanathan S, Dunn WB, Harrigan GG, Kell DB. Metabolomics by numbers: Acquiring and understanding global metabolite data. Trend Biotechnol 2004;22:245-52.

3. Nicholson JK, Lindon JC. Systems biology: Metabonomics. Nature 2008;455:1054-6.

4. Shyur LF, Yang NS. Metabolomics for phytomedicine research and drug development. Curr Opin Chem Biol 2008;12:66-71.

5. Clayton TA, Lindon JC, Cloarec O, et al. Pharmaco-metabonomic phenotyping and personalized drug treatment. Nature 2006;440:1073-7.

6. Nicholson JK. Global systems biology, personalized medicine and molecular epidemiology. Mol Syst Biol 2006;2:52.

7. Li P, Yang LP, Gong YW. Application of systems biology technology in research of traditional Chinese medicine. Trad Chin Med 2009;29:152-7.

8. Lao YM, Jiang JG, Yan L. Application of metabonomic analytical techniques in the modernization and toxicology research of traditional Chinese medicine. Br J Pharmacol 2009;157:1128-41.

9. Zhao L, Nicholson JK, Lu A, et al. Targeting the human genomemicrobiome axis for drug discovery: Inspirations from global systems biology and traditional Chinese medicine. J Proteome Res 2012;11:3509-19.

10. Wang XJ, Zhang AH, Sun H. Future perspectives of Chinese medical formulae: Chinmedomics as an effector. Omics 2012;7/8(414).

11. Legg K. Metabolomics: Gaining insight into pain. Nat Rev Drug Discov 2012;11:188-9.

12. Roberts MJ, Schirra HJ, Lavin MF, et al. Metabolomics: A novel approach to early and noninvasive prostate cancer detection. Korean J Urol 2011;52:79-89.

13. Sun H, Han Y, Zhang AH, et al. UPLC-MS based metabolic profiling of the phenotypes of Acanthopanax senticosus reveals the changes in active metabolites distinguishing the diversities of the plant grown in northeast area of China. Chin J Natural Med 2012;10:109-206.

14. Luo SH, Wu SW, Fan G, et al. Application of ${ }^{1}$ HNMR-based metabolomics of the species differentiation and quality evaluation of traditional Chinese medicine and Tibetan medicine from various species. J Third Military Med University 2013;3:996-1000.

15. Choi MY, Chai C, Park JH, et al. Effects of storage period and heat treatment on phenolic compound composition in dried Citrus peels (Chenpi) and discrimination of Chenpi with different storage periods through targeted metabolomic study using HPLC-DAD analysis. J Pharm Biomedl Analysis 2011;54:638-45.

16. Ma C, Bi KS, Zhang M. Toxicology effects of morning glory seed in rat: A metabonomic method for profiling of urine metabolic changes. J Ethnopharmacol 2010;130:134.

17. Ma C, Bi K, Su D, et al. Serum and kidney metabolic changes of rat nephrotoxicity induced by morning glory seed. Food Chem Toxicol 2010;48:2988.

18. Liu Y, Huang R, Liu L, et al. Metabonomics study of urine from Sprague-Dawley rats exposed to Huang-yao-zi using ${ }^{1} \mathrm{H}$-NMR spectroscopy. J Pharm Biomed Anal 2010;52:136.

19. Yan G, Zhao Y, Deng P, et al. Investigation of toxicological effects of Shuanghuanglian injection in Beagle dogs by metabonomic and traditional approaches. Exp Biol Med ( May-wood) 2010;235:1356.

20. Wang H, Bai J, Chen G, et al. A metabolic profiling analysis of the acute hepatotoxicity and nephrotoxicity of Zhusha Anshen Wan compared with cinnabar in rats using $1 \mathrm{H}$ NMR spectroscopy. J Ethnopharmacol 2013;146:572.

21. Wang J, Yuan ZM, Li YX, et al. The study on the compatibility mechanism of Rhizoma coptidis and radix rehmanniae in treating type II diabetes based on GC - MS metabolomic. J Chin Material Med 2014;39:526.

22. Wishart D. Quantitative metabolomics using NMR. Trends Anal Chem 2007;12:001.

23. Lu X, Zhao XJ, Bai CM, et al. LC-MS-basedmetabonomics analysis. J Chromatogr B 2007;10:022.

24. AJ, Trygg J, Gullberg J, et al. Extraction andGC/MS analysis of the human blood plasmametabolome. Anal Chem 2005;77:8086-94
25. Ebbels TM. Prediction and classification of drug toxicity using probabilistic modeling of temporal metabolic data: The consortium on metabonomic toxicology screening approach. J Proteome Res 2007;6:4407.

26. Li J, Wu XJ, Liu CX, Yuan Y. Application of new method for data processing in metabonomic studies. Acta Pharm Sin 2006;41:47-53.

27. Ding HT. The research of composition-activity relationship of curcuma volatile oil. Tianjin: Tianjin University 2012:1-54.

28. Wu HW, Chen JX, Yang HJ, et al. The relationship between compositions of salvia miltioorhiza and antioxidative capacity. Ch J Exp Trad Med Formulae 2009;15:68-71.

29. Chen C. Research on the the identification of effective components in essential oil of ligusticum chuanxiong hort based on data mining. Xi'an: Xi'an Jiaotong University 2009.

30. Li ZK, Chen JX, Yang HJ, et al. The analysis between the combination of ingredient in Ligusticum wallichii and vascular activity relationship based on Least Angel Regressions, 2009 .

31. Li FM, Lu XM, Liu HP, et al. A pharmaco-metabonomic study on the therapeutic basis and metabolic effects of Epimedium brevicornum Maxim on hydrocortisone-induced rat using UPLC MS. Biomedical Chromatography 2007;21:397-405.

32. Zhang H. Study on the identification of antitumor ingredient in total turmeric extracts and the combination of various components based on Optimized Potential For Liquid Simulations. Tianjin: Tianjin University, 2013.

33. Yu Y, Yi ZB, Liang YZ. Validate antibacterial mode and find main bioactive components of traditional Chinese medicine Aquilegia oxysepala. Bioorganic \& Medicinal Chemistry Letters 2007;17:1855-9.

34. Yi ZB, Yu Y, Liang YZ. Investigation of antimicrobial model of Hemsleya pengxianensis W.J.Chang and its main active component by metabolomics techniqu. J Ethnopharmacol 2008;116:89-95.

35. Yu Y, Yi ZB, Liang YZ. Main antimicrobial components of Tinospora capillipes, and their mode of action against Staphylococcus aureus. FEBS Letters 2007;581:4179-83.

36. Wang XJ, Li ZY, et al. Quality control over different processed products of Polygalae Radix based on plant metabolomics. Chin Trad Herbal Drugs 2012;42:1727-37.

37. Che QM, Huang XL, et al. Study on the drug metabolites of baicalin. J Chin Material Med 2001;26:768-9.

38. Wang Y. Study on the metabolism of baicalin in rat and the biological activity of it`s metabolite. Liaoning: Shenyang pharmaceutical university 2004: 1-92.

39. Lu YH, Wang GJ, Huang Q, et al. Investigations into metabonomic profiling of spontaneously hypertensive Rat ( SHR ) and metabolic effects of total ginsenoside on SHR using GC / MS. Chin J Nat Med 2007;5:443.

40. Gu Y, Zhang Y, Shi X, et al. Effect of traditional Chinese medicine berberine on type 2 diabetes based on comprehensive metabonomics. Talanta 2010;81:766.

41. Meng XS, Jiang M, Luo GA, et al. Mechanism research of rhizoma ligustici wallichii on haemorrheological nature blood stasis rats based on metabonomics. Liaoning Journal of Traditional Chinese Medicine 2012;39:218-20.

42. Yang QW, Yang L, et al. The initial study of UPLC-MS metabolomics on the anti-inflammatory effects of Radix paeoniae rubra and radix paeoniae alba. China Journal of Chinese Materia Medica 2012;36(6).

43. Li CY, Wang P, Wang Z, Zhang Y, MENG XL. Mechanism study of Rheum officinale on chronic renal failure based on metabonomics. Chinese Traditional and Herbal Drugs 2012;43:312-5.

44. Wang L, Song M, et al. Metabonomic study on the effects of allicin on rats. Acta Pharmaceutica Sinica 2009; 44:1019-24.

45. Gong M, Ye WH, et al. Metabonomic study of intervention effects of Morinda officinalis on 'Kidney-yang deficiency syndrome'. China Journal of Chinese Materia Medica 2012;37:1682-5.

46. Zhang SS, Liu XY, et al. Metabonomic study on protective effect of ethanol extracts of Drynariae Rhizoma on osteoporosis in rats urine by using UPLC-MS /MS. China Journal of Chinese Materia Medica 2012;37:658-62.

47. Lu X, Xiong Z, Li J, et al. Metabonomic study on 'Kidney-Yang Deficiency syndrome' and intervention effects of Rhizoma Drynariae extracts in rats using ultra performance liquid chromatography coupled with mass spectrometry. Talanta 2011;83:700. 
48. Wang DQ, Li XW, et al. Antipyretic effect of Bupleurum scorzonerifolium based on GC-MS metabonomics. Chinese Traditional and Herbal Drugs 2013;44:574-80.

49. Gu JN, Niu J, et al. Research on the action mechanism of Ginseng total saponins treating rats with diabetic cardiomyopathy by urine metabolism. Chinese J Anal Chem 2013; 413:371-6.

50. ZY Y, FY L, XB, et al. Ultra performance liquid chromatographybased metabonomic study of therapeutic effect of the surface layer of Poria cocos on adenine-induced chronic kidney disease provides new insight into anti-fibrosis mechanism. PLoS One 2013.

51. Qiao X, Ye M, Xiang C, et al. Metabolic regulatory effects of licorice: a bile acid metabonomic study by liquid chromatography coupled with tandem mass spectrometry. Steroids 2012;77:745.

52. Qi Y, Qu L, Wu Y, et al. A plasma metabonomic investigation into the intervention of volatile oil of Magnolia biondii pamp on rat model of acute inflammation. J Ethnopharmacol 2011;137:487.
53. Cao WL. Study on the mechanism of protecting liver and metabolic in vivo of total glucosides of paeony based on metabonomics. Hebei: hebei medical university 2014:1-84.

54. Liu P, Liu S, Tian D et al. The applications and obstacles of metabonomics in traditional Chinese medicine. Evid Based Complement Alternat Med, 2012, doi: 10. 1155 /2012/945824.

55. Qin KM, Wang B, et al. Perspective and application of metabonomics in modern study of traditional Chinese medicine. China Journal of Chinese Materia Medica 2014;39:3010-7.

56. Yang ZL, Hu QX, et al. The application progress, problems and strategies of metabonomics in Traditional Chinese medicine field. China science and technology of Chinese medicine 2014;21:715-7. 